\section{Severe asthma}

\section{M1 CONCORDANCE TO ASTHMA MEDICATIONS IN PATIENTS RECEIVING LONG-TERM OMALIZUMAB TREATMENT}

MS Elsherbiny, V Mitchell, AH Mansur, L Afridi, B Ahitan; Severe and Brittle Asthma Unit (SBAU) at Heartlands Hospital, Heart of England NHS Foundation Trust, Birmingham, United Kingdom

\subsection{6/thoraxjnl-2013-204457.411}

Introduction It is estimated that $5 \%$ of adult asthma patients have uncontrolled asthma despite high dose corticosteroid therapy. Omalizumab is a monoclonal anti-IgE antibody that has been shown to improve asthma control. ${ }^{1}$ As asthma control improves, concordance to other prescribed asthma medication may decline which may undermine omalizumab effectiveness. The extent of such non-concordance is explored in this study. Methods The records of 40 patients receiving long-term omalizumab treatment have been reviewed for concordance. Medication history was retrieved for all patients, which included all prescriptions issued by their General Practitioner over most recent twelve months, detailing the doses and the number of tablets and/or inhalers patients received. Patients were classified as non-concordant if their medication history showed less than $75 \%$ adherence. Concordant and nonconcordant groups were compared in relation to asthma control questionnaire (ACQ), Fractional exhaled nitric oxide (FeNO), forced expiratory volume in one second (FEV1), and number of asthma related admissions and frequency of severe steroid requiring asthma exacerbations at baseline line of omalizumab treatment.

Results Sufficient information was only available for 25 patients (20 female) receiving long-term omalizumab treatment with a mean age of 44 years (range 19 to 68 years) and mean weight of $78 \mathrm{~kg}(\mathrm{SD}=20.78)$. The prevalence of non-concordance was $64 \%(\mathrm{n}=16)$. All non-concordant patients had $50 \%$ or less prescription filling history. Overall non-concordant patients had a higher FEV1 before starting omalizumab treatment (mean FEV1 $=1.865,95 \%$ predicted) compared to concordant patients (mean FEV1 $=1.401,95 \%$ predicted), better ACQ scores (mean $=2.26$ ), less number of patients on oral steroids $(56.25 \% \mathrm{n}=9)$ compared to $(66.7 \% \mathrm{n}=6)$.

Conclusion The prevalence of non-concordance in patients receiving long-term omalizumab treatment is high and may undermine treatment effectiveness. The non-concordant patients had overall less severe asthma than concordant patients. Further research is needed to determine the effect of such non-concordance on the effectiveness of omalizumab in the long term.

\section{REFERENCE}

1. Efficacy and safety of a recombinant anti-immunoglobulin $E$ antibody (omalizumab) in severe allergic asthma. Holgate ST, Omalizumab 011 International Study Group.

\section{M2 BONE MINERAL DENSITY CHANGES IN PATIENTS IN THE SEVERE ASTHMA CLINIC ON ORAL CORTICOSTEROIDS}

HGT Brice, DJ Wilkinson, R Daly, G Tavernier, RM Niven; UHSM, Manchester, England

\subsection{6/thoraxjnl-2013-204457.412}

Objectives How effective are bone sparing medications (BSM)? Is bone mineral density (BMD) change related to other clinical variables?

Background Regular oral corticosteroids (OCSs) cause several side effects including BMD loss, occurring at varying rates in different patients.
Method Patients attending the specialist severe asthma clinic with two available bone densitometry scans since 2007 were identified and the hip and spine BMD change calculated. The cumulative steroid dose (pre and post first scan) and risk factors for osteoporosis were also recorded, including current bone protection.

Results Yearly hip BMD change and average cumulative steroid dose did not correlate for all patients $(\mathrm{n}=59$ Pearson $=-0.083$ $\mathrm{p} 0.53)$ or for patients not on BSM $(\mathrm{n}=27$ Pearson $=-0.26$ p0.19). Yearly spine BMD change also did not correlate for all patients $(\mathrm{n}=59$ Pearson $=0.012 \mathrm{p} 0.19)$ or for patients not on BSM $(\mathrm{n}=27$ Pearson $=-0.22 \mathrm{p} 0.28)$.

Body mass index (BMI) predicted a higher $\mathrm{t}$-score on the first DXA scan; hip $(\mathrm{n}=59)$ (Pearson $=0.59 \mathrm{p}<0.001)$ and spine $(\mathrm{n}=59)($ Pearson $=0.53 \mathrm{p}<0.001)$. However between scans, although not statistically significant, a higher BMI correlated with a greater decrease in BMD.

Analysing the effect of BSM (see figure: spine BMD change) showed spine BMD improved over time for patients taking a bisphosphonate $(\mathrm{n}=59) \mathrm{p} 0.029$. However when compared to patients on calcium/vitamin D or no BSM, patients on a bisphosphonate and calcium/vitamin $\mathrm{D}$ had a greater improvement $(+1.5 \%$ average BMD change, p0.039) than patients on a bisphosphonate alone $(+1.19 \%$ average BMD change, $\mathrm{p} 0.21)$. Conclusion OCS use causes a decrease in BMD which is greater in the spine. In this small population, cumulative steroid dose did not correlate to BMD change over time. Bisphosphonate and calcium/vitamin D demonstrated benefit in spine BMD between scans, whereas the benefit of bisphosphonate alone was not statistically significant.

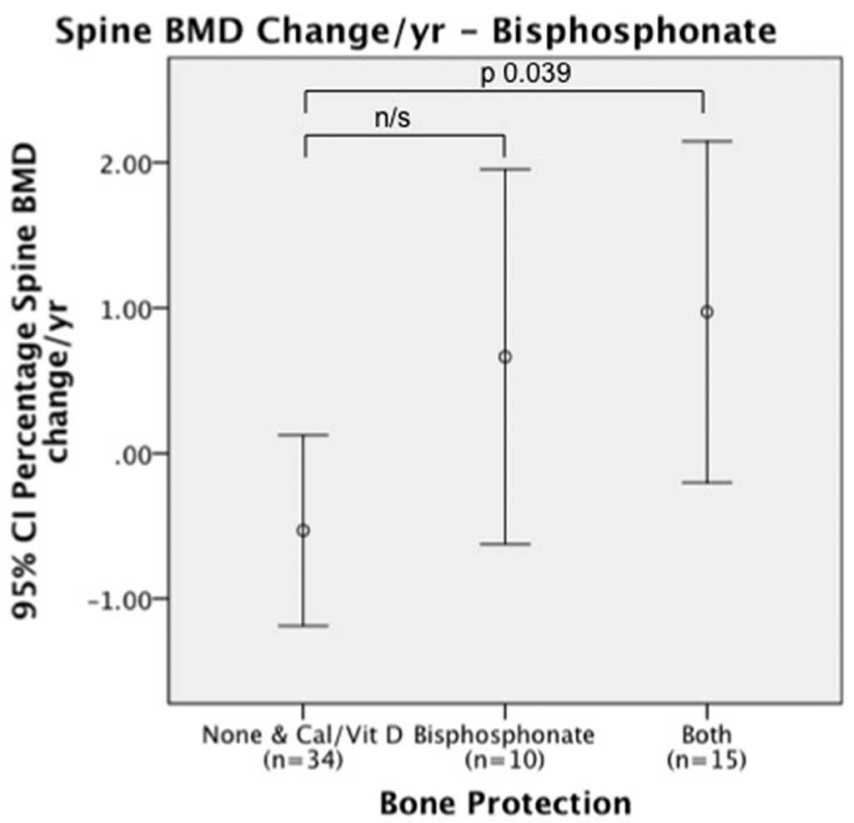

Abstract M2 Figure 1.

\section{M3 EVALUATION OF PHYSICAL ACTIVITY IN PATIENTS WITH SEVERE ASTHMA-ARE WE GETTING IT RIGHT?}

LF Grillo, S Greenwood, J Hull, A Menzies Gow; The Royal Brompton NHS Foundation Trust, London, UK

\subsection{6/thoraxjnl-2013-204457.413}

Introduction Of the 5 million patients who receive medication for asthma approximately $5 \%$ have severe or difficult to treat 
disease. A key problem for patients with severe asthma is impaired exercise capacity and often reported as a trigger for symptoms. In COPD it is recognised that reduced Physical Activity (PA) levels can be linked with poorer morbidity and increased exacerbations. In Severe Asthma (SA) there has to date been no formal evaluation of self reported PA levels in comparison to objective measurements and Quality of Life (QoL).

Objectives To evaluate self reported PA in a population of patients with SA compared to 6 Minute Walk Test (6MWT) results.

To compare PA levels with others measures of disease including lung function and QoL measurements (Short Form 36 or SF36)

Methods 35 patients (12 male:17 Female) with SA completed the Dukes Activity Status Index (DASI) which gives an estimated maximum metabolic equivalent (METs) as an estimation of self reported physical function. QoL was measured with the SF36 where Physical component scores (PCS) and Mental Component Scores (MCS) were collected. Objective measures of Forced Expiratory Volume in 1 second percent predicted $\left(\mathrm{FEV}_{1} \%\right)$ and 6MWT distance (metres) were also collected.

\section{Results}

\begin{tabular}{ll} 
Abstract M3. Table of demographics & \\
\hline & Median (range) \\
Age & $45(52)$ \\
FEV $\%$ & $68.1(87.4)$ \\
6MWT(metres) & $390(650)$ \\
SF36 PCS & $33.1(42.1)$ \\
SF36 MCS & $50.2(49.4)$ \\
DASI & $22.3(51)$ \\
\hline
\end{tabular}

The 6MWT had medium correlations with SF36 MCS $\left(r_{s}=0.372, p>0.03\right)$ and SF36 PCS $\left(r_{s}=0.480, p>0.004\right)$ and a strong correlation with the DASI $\left(r_{s}=0.604, p>\right.$ 0.0001). Moreover the Physical function component of SF36 (SF36PF) also had a strong correlation with 6MWT $\left(\mathrm{r}_{\mathrm{s}}=0.541\right.$, $\mathrm{p}>0.001) . \mathrm{FEV}_{1}$ showed a medium correlation with $6 \mathrm{MWT}$ $\left(\mathrm{r}_{\mathrm{s}}=0.413, \mathrm{p}>0.19\right)$.

Conclusion Patients appear to estimate PA levels accurately with the DASI, with higher scores of estimated METs correlating with increased 6MWT distance. QoL appears higher in those patients who achieve a greater distance in their 6MWT, especially in relation to their physical function. $\mathrm{FEV}_{1}$ preservation also appears to correlate with improved 6MWT distances. Further investigation of PA in this popoulation is warranted.

\section{M4 THE IMPACT OF AN ASTHMA INREACH SERVICE ON ASTHMA MANAGEMENT AND FOLLOW UP}

L Taylor, B Castell, J Willis-Chan, M O'Shea, M Masoli; Plymouth Hospitals NHS Trust, Plymouth, UK

\subsection{6/thoraxjn-2013-204457.414}

Introduction Suboptimal discharge management of asthmatic patients increases risk of early re-hospitalisation leading to avoidable burden on healthcare services. Previous BTS asthma audits have demonstrated that discharge planning was an area for improvement. In Sept 2011 we developed an asthma Inreach team ( 2 band 6 nurses, 1.3 whole time equivalents) to improve asthma inpatient care. In Oct 2012 this service was expanded to actively trace all patients who had attended the Emergency
Department (ED) in the previous $24 \mathrm{hrs}$ and arrange follow up in an asthma nurse specialist clinic (ANS) within one week. We evaluated the impact of this service.

Methods Data was extracted from clinic letters. The asthma Inreach team reviewed patients admitted to the respiratory wards and a proforma was used to document interventions.

Results ED: Between Oct 2012 to May 2013, there were 215 patients with 245 attendances to ED. 12 patients were readmitted within 30days. Only $26(12 \%)$ patients were known to chest clinic. 138 (64\%) attended follow up in the ANS clinic. 70 (51\%) had poor compliance issues addressed. 48 (22\%) had a significant change in treatment and $30(14 \%)$ were referred onto the difficult asthma clinic. All received written asthma management plans (WAMP). Asthma Inreach: Between Sept 2011 to May 2013, the asthma inreach team undertook 240 reviews on 213 patients. $142(68 \%)$ patients given WAMP 91 (43\%) inhaler technique initially poor improved with education. 65 (27\%) had poor compliance. 155 (73\%) patients were subsequently followed up in ANS clinic. 30 day readmission rate decreased from $2.7 \%$ in $2011 / 12$ to $1.9 \%$ in $2012 / 13$.

Conclusion This study demonstrates that the introduction of an asthma inreach service \& ANS clinic has significantly improved asthma management, follow up according to BTS guidelines and reduced 30 day readmissions. A significant number of patients had evidence of poor compliance, poor inhaler technique, lack of WAMP which was addressed by the asthma team. A number of patients were more complex and required review in the difficult asthma clinic.

\section{M5 LUNG FUNCTION AND PSYCHOLOGICAL WELL-BEING: ONE-YEAR OUTCOMES IN SEVERE ASTHMA}

R Satherley, J Fellows, V Mitchell, AH Mansur; Severe and Brittle Asthma Unit, Heartlands Hospital, Birmingham, England

\subsection{6/thoraxjnl-2013-204457.415}

Aims Psychological comorbidity and impaired quality of life (QoL) are commonly reported in severe and difficult to treat asthma. These factors appear to contribute to asthma attacks and hospital admissions. Changes in these markers over one-year were studied following multidisciplinary intervention.

Research Design and Methods All new referrals to the Severe and Brittle Asthma Unit (SBAU), between 2009 and 2012 were assessed using a structured protocol including asthma diagnosis and severity, associated co-morbidities, lung function, QoL and drivers of poor asthma control. Patients received multidisciplinary interventions over a one-year period and outcomes were reassessed. Measures used included the Hospital Anxiety and Depression Scale (HADS) and the Juniper QoL. Lung function measures included $\mathrm{FEV}_{1}$ and the Asthma Control Questionnaire. Paired T-Tests and Multiple Regressions were used to analyse the data.

Results 142 patients $(\mathrm{N}=108,76.06 \%$ female) completed the baseline measures. After one-year of multidisciplinary intervention 66 completed the HADS, 69 completed the Juniper QoL and 102 had $\mathrm{FEV}_{1}$ reassessed. The majority $(\mathrm{N}=96 ; 85.71 \%)$ of these patients met the criteria for refractory asthma. The number of patients on antidepressants significantly decreased over the year from $17.61 \%(\mathrm{~N}=25)$ to $10.71 \%(\mathrm{~N}=12$; $\mathrm{p}=0.021)$. Depression on the HADS showed a non-significant reduction from $53.5 \%(\mathrm{~N}=76)$ to $42.4 \%(\mathrm{~N}=28$; $\mathrm{p}=.192)$ after one year. Anxiety on the HADS showed clinically significant reduction from $57.7 \%(\mathrm{~N}=82)$ to $40.9 \%$ 